\title{
Schönheitsoperation, damit das Selfie besser aussieht?
}

Einst suchten Menschen den kosmetischen Chirurgen in der Hoffnung auf, ihr reales Aussehen zu verbessern. Inzwischen aber geht es vielen vor allem um ihre virtuelle Schönheit: auf dem Selfie.

Du sollst dir kein Bildnis machen - dieses Gebot mag in biblischen Zeiten gegolten haben. Das ist allerdings schon ein paar Jahre her. Jedenfalls gebietet die Gegenwart offenbar das Gegenteil: Du sollst so viele Bildnisse von dir machen wie nur möglich, und die Resultate sollst du in den sozialen Medien präsentieren.

Freilich hat, wie jeder Götzendienst, auch die Selfiemanie ihre Nachteile. Einer davon ist, dass die Arme für den Schnappschuss vom eigenen Gesicht für den Anspruch ans eigene Aussehen oft zu kurz gewachsen sind. Das führt zu Verzerrungen speziell im Mittelgesichtsbereich, sodass die Nase zu überproportionaler Größe angeschwollen erscheint (• Abb. 1). Boris Paskhover, Spezialist für Gesichtschirurgie an der Rutgers New Jersey Medical School in Newark, hat zusammen mit Kollegen ausgerechnet, dass ein durchschnittli-

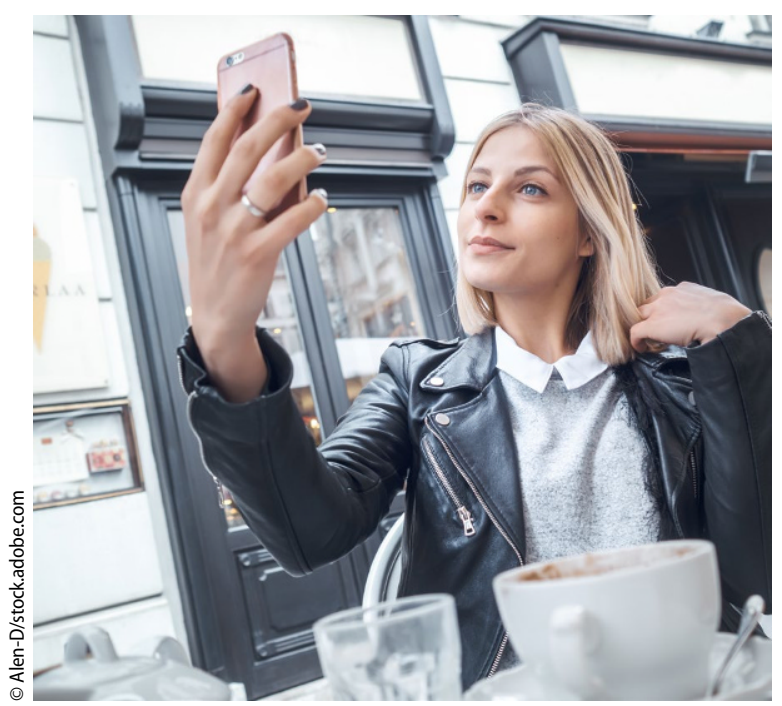

Abb. $1 \Delta$ Weil die Arme nicht lang genug sind, wirkt die Nase auf Selfies zu groß. (Symbolbild mit Fotomodell) ches Selfie die Nasenspitze um $7 \%$ und die Nasenbasis um $30 \%$ gegenüber einer Aufnahme in einem Porträtabstand von circa eineinhalb Metern vergrößert [1].

\section{Komik mit ernsten Folgen}

Solche Porträts mögen vielleicht komisch aussehen, haben aber mitunter ernste Konsequenzen. Laut Angaben der American Academy of Facial Plastic and Reconstructive Surgeons berichten $55 \%$ der plastischen Chirurgen von Patienten, die zu ihnen gekommen seien, um sich zur Verbesserung der Selfieanmutung einer kosmetischen Operation zu unterziehen.

Dabei handelt es sich offenbar nicht um ein flüchtiges Phänomen. Eine aktuelle Studie bestätigt vielmehr den Zusammenhang der Nutzung sozialer Medien inklusive Applikationen zur Bildbearbeitung mit einer gesteigerten Akzeptanz kosmetisch-chirurgischer Eingriffe. Eine Arbeitsgruppe unter Leitung von Dr. Lisa Ishi, HNO-Abteilung der Johns Hopkins University School of Medicine, hat dafür 252 Teilnehmer webbasiert befragt [2].

Es zeigte sich, dass die Akzeptanz ästhetischer Eingriffe durch folgende Merkmale erhöht wird: Nutzung von YouTube, Tinder sowie Snapchat mit oder ohne Filter; höhere Abhängigkeit des Selbstwertgefühls vom eigenen Erscheinungsbild; und den Umstand, dass die Betreffenden schon einmal ein Bild von sich aus sozialen Medien entfernt haben, weil es nicht bis zur Zufriedenheit digital bearbeitet oder verbessert worden war. Auch gaben Menschen, die viel Zeit und Energie in soziale Medien investierten, Instagram-Fotofilter oder die App der Visual Supply Company (VSCO) nutzten, zu Protokoll, sich stärker mit den Möglichkeiten kosmetischer Chirurgie zu befassen.

Billiger und der körperlichen Integrität dienlicher wäre es vermutlich, dem biblischen Rat zu folgen und wenn schon nicht keine, so doch zumindest erheblich weniger Bildnisse anzufertigen.

\section{Literatur}

1. Ward B et al. (2018) Nasal Distortion in Short-Distance Photographs: The Selfie Effect. JAMA Facial Plast Surg 20:333-5. https://doi.org/10.1001/jamafacial.2018.0009

2. Chen J et al. (2019) Association Between the Use of Social Media and Photograph Editing Applications, Self-esteem, and Cosmetic Surgery Acceptance. JAMA Facial Plast Surg;21:361-367. https://doi.org/10.1001/ jamafacial.2019.0328

hautnah 2020 · 19:18

https://doi.org/10.1007/s12326-01900354-w

C Springer-Verlag GmbH Austria, ein Teil von Springer Nature 2019 